\title{
LETTER
}

\section{High prevalence of acute stress disorder and persisting symptoms in ICU survivors after COVID-19}

\author{
Silvia Mongodi ${ }^{*} \mathbb{D}$, Giulia Salve ${ }^{2}$, Guido Tavazzi ${ }^{1,2}$, Pierluigi Politi ${ }^{3}$, Francesco Mojoli ${ }^{1,2}$ on behalf of the \\ COVID-19 Post-ICU team and COVID-19 Pavia Crisis Unit
}

@ 2021 Springer-Verlag GmbH Germany, part of Springer Nature

Dear Editor,

Post-traumatic stress disorder may surge following exposure to life-threatening events and affects around $20 \%$ of intensive care unit (ICU) survivors [1, 2]; a higher rate $(38.8 \%)$ was observed in Middle East and Severe Acute Respiratory Syndromes' outbreaks [3]. The coronavirus disease 19 (COVID-19) pandemic [4] presents all the features to deeply impact not only on physical but also on mental health: patients are isolated from their families, communication with healthcare providers is difficult because of personal protective equipment and discrepancy between patients' number and staff members is evident. A significant psychological impact on general population and healthcare providers was demonstrated [5].

In a follow-up hospital visit, 47 patients (males $70.2 \%$, 59 [50-66] year-old) admitted to our ICU for COVID-19 from February 21st to June 5th, 2020 (Fig. 1 ESM) were assessed in person by an intensivist at least 1 month after hospital discharge [median time 72 [55-92] days). Informed consent was collected following the ad hoc procedures defined by the Ethics Committee for COVID-19 pandemic. The Impact of Event Scale-Revised (IES-RTable 1 ESM) was used to screen for acute stress disorder (ASD), defined by a score $\geq 33$. Median IES-R score was 30 [19-41]; 19 patients (40.4\%) showed ASD. Among subscales, intrusion average score was higher than avoidance $(1.6[1-2.4]$ vs. $1.25[0.8-1.8] ; p=0.0660)$; both

\footnotetext{
*Correspondence: silvia.mongodi@libero.it

${ }^{1}$ Anesthesia and Intensive Care, Rianimazione I, Fondazione IRCCS Policlinico S. Matteo, DEA piano -1, Viale Golgi 19, 27100 Pavia, Italy Full author information is available at the end of the article Silvia Mongodi and Giulia Salve equal contribution.
}

were higher than hyperarousal (0.9 [0.3-1.3]; $p<0.0001$ and $p=0.0114)$. No significant difference in patients' characteristics, length of stay in ICU/hospital, need and length of invasive/non-invasive ventilation and drugs was observed comparing ASD and non-ASD patients (Table 1). ASD patients were less hypoxemic at ICU admission; we can just speculate that, being less dyspnoeic, they possibly had more clear-headed experiences of their own condition and of the surrounding environment, including other patients' struggle and death. Patients with IES-R score higher than 30 were addressed to outpatients' facilities of their catchment area, to receive an accurate diagnosis and psychotherapy/medication, if necessary.

Anosmia, ageusia, altered hearing and balance persisted in $10.6 \%, 19.2 \%, 10.6 \%$ and $19.2 \%$, respectively. Asthenia and dyspnoea were scored with a 1-5 range. Asthenia persisted in $97.8 \%$ of patients (median score 2 [1-3]). Four patients (8.5\%) complained of dyspnoea at rest, two required home oxygen therapy. Patients complaining of dyspnoea for mild, moderate and severe efforts were 13 (27.7\%), 46 (97.8\%) and 46 (97.8\%), respectively. The number of patients complaining of dyspnoea in each of these classes did not differ significantly in ASD and non-ASD patients; however, dyspnoea score was higher in ASD patients (Table $2 \mathrm{ESM}$ ).

Most of the patients had unclear memories of ICU stay; the most common complaint was the feeling of complete isolation due to limited possibility to communicate with families/physicians. Many patients described nightmares, feeling of derealization and the initial belief of being in a fake hospital; this mainly affected the first patients of the pandemic. 10 patients (21.3\%-ASD 4 (21.1\%) vs.

\section{实


Table 1 Features of the patients discharged from ICU after COVID-19 with and without acute stress disorder

\begin{tabular}{|c|c|c|c|c|}
\hline & Overall $(n=47)$ & No ASD $(n=28)$ & ASD $(n=19)$ & $p$ value \\
\hline Age-years, median [IQR] & $59[50-66]$ & $60.5[50.5-67]$ & $57[50-65]$ & 0.4540 \\
\hline Sex-males, $n(\%)$ & $33(70.2)$ & $22(78.6)$ & $11(57.9)$ & 0.195 \\
\hline $\mathrm{BMI}-\mathrm{kg} / \mathrm{m}^{2}$, median [IQR] & $27.7[25.4-30.7]$ & $27.3[25.4-31]$ & $27.7[25.1-30.7]$ & 0.5543 \\
\hline SAPS II-median [IQR] & $31[27-45]$ & $34[26-48]$ & $30.5[27-39.5]$ & 0.3109 \\
\hline Past medical history of psychiatric diseases- $n$ (\%) & $2(4.3)$ & $0(0.0)$ & $2(10.5)$ & 0.079 \\
\hline $\mathrm{FiO}_{2}$ at ICU admission-median [IQR] & $0.8[0.6-1]$ & $1[0.7-1]$ & $0.6[0.6-1]$ & 0.0734 \\
\hline $\mathrm{PaO}_{2} / \mathrm{FiO}_{2}$ at ICU admission-mmHg, median [IQR] & 123 [84-159] & $100[82-130]$ & 142 [121-195] & 0.0114 \\
\hline Length of stay in hospital before ICU-days, median [IQR] & $3[1-8]$ & $3[1.5-7.5]$ & $3[0-8]$ & 0.6942 \\
\hline Length of stay in ICU-days, median [IQR] & 24 [10-37] & $25.5[11-40.5]$ & 19 [9-37] & 0.2828 \\
\hline Length of stay in hospital after ICU-days, median [IQR] & $9[6-15]$ & $9[6-14]$ & $12[6-21]$ & 0.4712 \\
\hline Length of CPAP before ICU admission-days, median [IQR] & $2[1-5]$ & $3[1-5]$ & $1[1-8]$ & 0.6927 \\
\hline Invasive mechanical ventilation in ICU-n (\%) & $34(72.3)$ & $21(75)$ & $13(68.4)$ & 0.621 \\
\hline Length of IMV-days, median [IQR] & $17.5[9-28]$ & $17[11-30]$ & $18[7-25]$ & 0.3293 \\
\hline Length of NMBA-days, median [IQR] & $7[3.5-11.5]$ & $7[6-12]$ & $5[3-11]$ & 0.2163 \\
\hline Pronation- $n(\%)$ & $19(40.4)$ & $11(39.3))$ & $8(42.1)$ & 0.773 \\
\hline Benzodiazepine sedation in ICU- $n(\%)$ & $23(48.9)$ & $16(57.1)$ & $7(36.8)$ & 0.238 \\
\hline Dexmedetomidine sedation in ICU- $n$, (\%) & $23(48.9)$ & $13(46.4)$ & $10(52.6)$ & 0.770 \\
\hline
\end{tabular}

$A S D$ acute stress disorder; IQR Interquartile range; BMI Body mass index; SAPS II Simplified acute physiology score; CPAP continuous positive airways pressure; ICU intensive care unit; $P E E P$ positive end-expiratory pressure; $\mathrm{PaO} 2$ arterial partial oxygen pressure; $\mathrm{FiO}_{2}$ fraction of inspired oxygen; $I M V$ invasive mechanical ventilation; NMBA neuromuscular blocking agents

non-ASD patients 6 (21.4\%); $p=0.975$ ) wanted to see the ICU, both to meet the ICU staff and to help their memories' reconstruction process.

In conclusion, ASD prevalence is high among ICU survivors after COVID-19; a systematic screening should be performed to reduce chronic effects of critical illness. Symptoms of asthenia and dyspnoea for moderate efforts persist for weeks after ICU and hospital discharge in a large majority of the patients.

\section{Supplementary Information}

The online version contains supplementary material available at https://doi. org/10.1007/s00134-021-06349-7.

\section{Author details}

${ }^{1}$ Anesthesia and Intensive Care, Rianimazione I, Fondazione IRCCS Policlinico S. Matteo, DEA piano -1, Viale Golgi 19, 27100 Pavia, Italy. ${ }^{2}$ Department of Clinical-Surgical, Diagnostic and Pediatric Sciences, Unit of Anesthesia and Intensive Care, University of Pavia, Pavia, Italy. ${ }^{3}$ Department of Brain and Behavioral Sciences, University of Pavia, Via Bassi 21, 27100 Pavia, PV, Italy.

\section{Acknowledgements}

COVID-19 Post-ICU team: Andrea Stella (San Matteo Hospital, Pavia, Italy), Andrea Colombo (San Matteo Hospital, Pavia, Italy), Valentino Dammassa (Department of clinical-surgical, diagnostic and pediatric sciences, Unit of anesthesia and intensive care, University of Pavia, Pavia, Italy), Sandro Pregnolato (Department of clinical-surgical, diagnostic and pediatric sciences, Unit of anesthesia and intensive care, University of Pavia, Pavia, Italy), Silvia Bonaiti (Department of clinical-surgical, diagnostic and pediatric sciences, Unit of anesthesia and intensive care, University of Pavia, Pavia, Italy).

COVID-19 Pavia Crisis Unit: Marena Carlo (San Matteo Hospital, Pavia, Italy); Calvi Monica (San Matteo Hospital, Pavia, Italy); Grugnetti Giuseppina (San Matteo Hospital, Pavia, Italy); Maurelli Marco (San Matteo Hospital, Pavia, Italy);
Muzzi Alba (San Matteo Hospital, Pavia, Italy); Raffaele Bruno (San Matteo Hospital, Pavia, Italy); Lago Paolo (San Matteo Hospital, Pavia, Italy); Marseglia Gianluigi (San Matteo Hospital, Pavia, Italy); Perlini Stefano (San Matteo Hospital, Pavia, Italy); Palo Alessandra (San Matteo Hospital, Pavia, Italy); Baldanti Fausto (San Matteo Hospital, Pavia, Italy); Oltrona Visconti Luigi (San Matteo Hospital, Pavia, Italy); Corsico Angelo Guido (San Matteo Hospital, Pavia, Italy); Di Sabatino Antonio (San Matteo Hospital, Pavia, Italy); Mojoli Francesco (San Matteo Hospital, Pavia, Italy); lotti Giorgio (San Matteo Hospital, Pavia, Italy); Benazzo Marco (San Matteo Hospital, Pavia, Italy); Carlo Nicora (San Matteo Hospital, Pavia, Italy); Antonio Triarico (San Matteo Hospital, Pavia, Italy); Vincenzo Petronella (San Matteo Hospital, Pavia, Italy), Davide Piloni (San Matteo Hospital, Pavia, Italy), Marco Vincenzo Lenti (First Department of Medicine, Fondazione IRCCS Policlinico S. Matteo, Pavia, Italy), Sara Cutti (Medical Direction, Fondazione IRCCS Policlinico S. Matteo, Pavia, Italy).

\section{Author contributions}

SM, GS, GT, FM actively contributed to the conception of the study, data collection and analysis; all the authors actively contributed to the redaction of the draft, provided critical revision of the article and provided final approval of the version submitted for publication.

\section{Funding}

Institutional funding.

\section{Compliance with ethical standards}

\section{Conflicts of interest}

FM received fees for lectures from GE Healthcare, Hamilton Medical, SEDA SpA, outside the present work. SM received fees for lectures from GE Healthcare, outside the present work. GT received fees for lectures from GE Healthcare, outside the present work. A research agreement is active between University of Pavia and Hamilton Medical. The other authors declare no conflict of interest.

\section{Informed consent}

All patients signed an informed consent for data publication. 


\section{Ethics approval}

Approved by local ethic committee.

\section{Consent to participate}

All patients signed an informed consent for data collection.

\section{Publisher's Note}

Springer Nature remains neutral with regard to jurisdictional claims in published maps and institutional affiliations

Accepted: 7 January 2021

Published online: 17 March 2021

\section{References}

1. ICD-11 International Classification of Diseases 11th Revision. The global standard for diagnostic health information. https://icd.who.int/en
2. Righy C, Rosa RG, Amancio T, da Silva R, Kochhann R et al (2019) Prevalence of post-traumatic stress disorder symptoms in adult critical care survivors: a systematic review and meta-analysis. Crit Care 23:213

3. Ahmed H, Patel K, Greenwood DC, Halpin S et al (2020) Long-term clinical outcomes in survivors of severe acute respiratory syndrome (SARS) and middle east respiratory syndrome (MERS) coronavirus outbreaks after hospitalisation or ICU admission: a systematic review and meta-analysis. J Rehabil Med 52(5):irm00063

4. Grasselli G, Greco M, Zanella A et al (2020) Risk factors associated with mortality among patients with COVID-19 in intensive care units in lombardy. Italy JAMA Intern Med 180(10):1345-1355

5. Chew NWS, Lee GKH, Tan BYQ et al (2020) A multinational, multicentre study on the psychological outcomes and associated physical symptoms amongst healthcare workers during COVID-19 outbreak. Brain Behav Immun 88:559-565 